\title{
Canada : l'école peut enseigner la religion, mais le conseil municipal ne peut prier
}

Stéphane Bernatchez et Marie-Pierre Robert

\section{OpenEdition}

Édition électronique

URL : http://journals.openedition.org/rdr/970

DOI : $10.4000 /$ rdr.970

ISSN : 2534-7462

Éditeur

Presses universitaires de Strasbourg

\section{Édition imprimée}

Date de publication : 8 novembre 2016

Pagination : 185-191

ISBN : 978-2-86820-959-7

ISSN : 2493-8637

Référence électronique

Stéphane Bernatchez et Marie-Pierre Robert, «Canada : l'école peut enseigner la religion, mais le conseil municipal ne peut prier », Revue du droit des religions [En ligne], 2 | 2016, mis en ligne le 11 février 2020, consulté le 10 décembre 2020. URL : http://journals.openedition.org/rdr/970 ; DOI : https://doi.org/10.4000/rdr.970

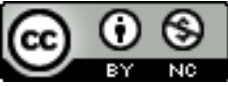

La revue du droit des religions est mise à disposition selon les termes de la Creative Commons Attribution - Pas d'Utilisation Commerciale 4.0 International - CC BY-NC 4.0. 


\section{CANADA : L'ÉCOLE PEUT ENSEIGNER LA RELIGION, MAIS LE CONSEIL MUNICIPAL NE PEUT PRIER}

\section{Stéphane BERNATCHEZ et Marie-Pierre ROBERT}

Centre de recherche Société, Droit et Religions de I'Université de Sherbrooke (SoDRUS)

— n 2015, la Cour suprême du Canada a rendu deux décisions qui retiennent El'attention en droit des religions : la première, en matière scolaire et la seconde, en matière municipale.

\section{L'AFFAIRE ÉCOLE SECONDAIRE LOYOLA}

Le 19 mars 2015, la Cour suprême du Canada a rendu une décision intéressante découlant d'une exemption demandée par un collège privé catholique au sujet du nouveau cours d'éthique et de culture religieuse ${ }^{1}$. Dans cette affaire, l'institution invoquait la restriction de sa liberté de religion, un argument qui impliquait que les personnes morales jouissent de la protection de cette liberté, ce qui n'était - et n'est toujours pas - clairement établi en droit canadien.

Dans la foulée de la déconfessionnalisation de l'enseignement religieux au Canada, le Québec a mis en place en 2008 un cours d'éthique et de culture religieuse neutre, qui remplace l'enseignement religieux ou moral qui était

1. École secondaire Loyola c. Québec (Procureur général), [2015] 1 R.C.S. 613 (ci-après Loyola). 
offert auparavant ${ }^{2}$. Ce cours, qui a pour objectifs la reconnaissance de l'autre et la poursuite du bien commun, comprend trois volets : les religions du monde et le phénomène religieux, l'éthique et le dialogue. Parmi les religions étudiées, on y voit le catholicisme, le protestantisme, le judaisme, l'islam, le bouddhisme, l'hindouisme, ainsi que les spiritualités autochtones. Ce cours est obligatoire pour tous les élèves, qu'ils soient inscrits à une école publique ou privée.

Le collège Loyola, un collège catholique anglophone tenu par les jésuites, a demandé au ministère de l'Éducation une exemption afin de pouvoir enseigner le catholicisme dans une perspective confessionnelle - et non neutre tel que le prévoit le programme. Le collège souhaitait également que la perspective catholique soit prédominante dans l'enseignement de l'éthique. Cette demande lui a été refusée, parce que, selon la ministre, le contenu du cours proposé n'était pas équivalent à celui du programme. La décision de la ministre a été contestée devant les tribunaux, et ce, jusqu'en Cour suprême du Canada.

La plus haute cour du pays a estimé que la décision ministérielle ne pouvait être maintenue, parce qu'elle ne respectait pas la liberté de religion. Pour en venir à cette conclusion, deux approches différentes ont été adoptées, qui se traduisent par deux énoncés de motifs différents : ceux de la majorité de quatre juges (les juges LeBel, Abella, Cromwell et Karakatsanis) et ceux, concurrents, de la minorité de trois juges (les juges McLachlin, Rothstein et Moldaver).

Tout d'abord, la majorité aborde la question sous l'angle du droit administratif ${ }^{3}$, en évaluant le caractère raisonnable de la décision ministérielle à l'aune d'une mise en balance des droits et libertés, d'une part, et du mandat de la loi applicable, d'autre part. Dans cette affaire, cette mise en balance implique la protection de la liberté de religion ainsi que les valeurs d'un État laĩque 4 . La minorité adopte une approche davantage basée sur le droit constitutionnel, en analysant la restriction d'une liberté, puis en appliquant la clause justificative.

La majorité estime, malheureusement, qu'il n'est pas nécessaire de trancher la question novatrice de savoir si une personne morale jouit de la

2. V. S.L. c. Commission scolaire des Chênes, [2012] 1 R.C.S. 235, un arrêt qui avait débouté des parents catholiques qui estimaient que le fait que leurs enfants soient exposés à d'autres religions dans le cadre de ce cours violait leur liberté de religion.

3. Selon le cadre d'analyse tracé dans Doré c. Barreau du Québec, [2012] 1 R.C.S. 395.

4. Loyola, par. 43. 
liberté de religion en droit canadien pour trancher l'affaire ${ }^{5}$. Elle estime que, compte tenu du caractère collectif de cette liberté, les institutions sont essentielles à l'épanouissement des membres qui la forment, soit, en l'occurrence, les enseignants, parents et élèves catholiques. C'est donc à travers le prisme de la liberté de religion individuelle que les motifs majoritaires sont rédigés. Les motifs font notamment référence à l'atteinte au droit des parents de transmettre leur foi catholique ${ }^{6}$ ainsi qu'à celui des professeurs d'enseigner la religion qui constitue l'identité même du collège au sein duquel ils œuvrent ${ }^{7}$.

La minorité adopte, quant à elle, l'approche tout à fait inverse en estimant que comme la liberté de religion de l'institution a été restreinte, il n'est pas nécessaire de se pencher sur la violation de la liberté des membres qui la composent ${ }^{8}$. La minorité estime donc que le collège Loyola, comme institution, jouit de la protection de la liberté de religion, puisqu'il s'agit d'une organisation religieuse, constituée principalement à cette fin et dont les activités sont compatibles avec celle-ci ${ }^{9}$. Cela semble impliquer que les personnes morales qui ne sont pas des organisations religieuses ne peuvent jouir de la liberté de religion, contrairement à ce qui a été décidé récemment en droit américain ${ }^{10}$. De plus, cette application de la liberté de religion à une personne morale implique des aménagements particuliers. En effet, comme la restriction de la liberté de religion s'analyse en fonction du critère de la croyance sincère $^{11}$, un critère difficilement applicable à une entité désincarnée, la minorité a adapté ce critère à la personne morale en référant plutôt à sa mission et à ses activités ${ }^{12}$.

Finalement, en ce qui concerne la réparation à apporter, alors que la majorité renvoie au ministre pour un réexamen, la minorité aurait ordonné au ministre d'octroyer l'exemption.

Suite à cet arrêt, d'autres collèges privés confessionnels pourront espérer obtenir une exemption ministérielle pour enseigner le cours d'éthique et culture religieuse dans leur perspective confessionnelle, quelle qu'elle soit.

5. Ibid., par. 34

6. Ibid., par. 64.

7. Ibid., par. 63.

8. Ibid., par. 130-131.

9. Ibid., par. 100.

10. Burwell v. Hobby Lobby, Inc., [2014] 573 US.

11. Syndicat Northcrest c. Amselem, [2004] 2 R.C.S. 551.

12. Loyola, par. 138-140. 


\section{L'AFFAIRE SAGUENAY ${ }^{13}$}

Le 15 avril 2015, la Cour suprême du Canada a décidé que la récitation de la prière lors des séances publiques de délibérations du conseil municipal de la Ville de Saguenay contrevient à l'obligation de neutralité qui incombe à l'État et constitue une atteinte discriminatoire à l'égard du citoyen athée qui avait porté plainte à la Commission des droits de la personne et des droits de la jeunesse du Québec. Cette pratique contraint à l'adhésion à certaines croyances religieuses alors que l'État est tenu d'agir dans le respect de la liberté de conscience et de religion de chacun. Le corollaire de cette liberté veut que l'État demeure neutre en matière religieuse.

Le maire, les conseillers et principaux fonctionnaires de la ville de Saguenay débutaient les séances publiques du conseil municipal par la récitation, par le maire, d'une prière, précédée et suivie d'un signe de croix accompagné des mots « au nom du Père, du Fils et du Saint-Esprit». De 2002 à 2008, aucun règlement ne régissait la prière, qui se lisait alors comme suit :

« Ô Dieu, éternel et tout puissant, de qui vient tout pouvoir et toute sagesse, nous voici assemblés en votre présence pour assurer le bien et la prospérité de notre ville. Accordez-nous, nous vous en supplions, la lumière et l'énergie nécessaires pour que nos délibérations soient destinées à promouvoir l'honneur et la gloire de votre saint nom et le bonheur spirituel et matériel de notre ville. Ainsi soit-il.»

Suite au dépôt de la plainte par le Mouvement laïque québécois au nom de monsieur Alain Simoneau, la Ville a adopté un règlement encadrant la prière et prévoyant un délai de deux minutes entre la fin de la prière et l'ouverture officielle des séances du conseil. Le texte de la prière a alors été modifié :

« Dieu tout puissant, nous Te remercions des nombreuses grâces que $\mathrm{Tu}$ as accordées à Saguenay et à ses citoyens, dont la liberté, les possibilités d'épanouissement et la paix. Guide-nous dans nos délibérations à titre de membre du conseil municipal et aide-nous à bien prendre conscience de nos devoirs et responsabilités. Accorde-nous la sagesse, les connaissances et la compréhension qui nous permettront de préserver les avantages dont jouit notre ville afin que tous puissent en profiter et que nous puissions prendre de sages décisions. Amen. »

Le Tribunal des droits de la personne, qui entendait l'affaire en première instance, a déterminé que la récitation de la prière constituait une atteinte

13. Mouvement laïque québécois c. Saguenay (Ville), 2015 CSC 16, [2015] 2 R.C.S. 3. 
discriminatoire et a déclaré en conséquence le règlement inopérant et sans effet, dans la mesure où il prescrivait un objet religieux qui contrevient à l'obligation de neutralité de l'État. La Cour d'appel du Québec a jugé, quant à elle, que la notion de neutralité n'oblige pas l'État à faire preuve d'abstentionnisme en matière religieuse. Retenant une notion de neutralité bienveillante, le juge Guy Gagnon, de la Cour d'appel, était d'avis que l'obligation de neutralité doit tenir compte de l'héritage et des traditions de la société, et se conjuguer avec le devoir de l'État de préserver son histoire et son patrimoine religieux.

La jugeant inconciliable avec l'obligation de l'État de demeurer neutre en matière religieuse, la Cour suprême du Canada a rejeté cette notion de neutralité bienveillante qui permettrait à l'État d'adhérer à une croyance religieuse : « $\mathrm{Si}$, sous le couvert d'une réalité culturelle, historique ou patrimoniale, l'État adhère à une forme d'expression religieuse, il ne respecte pas son obligation de neutralité. ${ }^{14}$ Selon la Cour suprême, «le parrainage par l'État d'une tradition religieuse, en violation de son devoir de neutralité, constitue de la discrimination à l'endroit de toutes les autres» : «Si l'État favorise une religion au détriment des autres, il crée une inégalité destructrice de la liberté de religion dans la société. ${ }^{15}$ Même si l'obligation de neutralité religieuse n'est pas explicitement prévue dans la Charte québécoise (ni dans la Charte canadienne d'ailleurs), elle découle de la liberté de conscience et de religion. Elle exige que l'État ne favorise ni ne défavorise aucune croyance, pas plus du reste que l'incroyance ${ }^{16}$. La Cour suprême se dit d'accord avec le Tribunal des droits de la personne qui avait décidé qu'une " autorité étatique ne peut instrumentaliser ses pouvoirs afin de promouvoir ou d'imposer une croyance religieuse $»^{17}$.

Selon la Cour, la pratique qui consiste à réciter la prière et le règlement qui l'encadre entraînent l'exclusion de M. Simoneau sur la base de la religion (qui comprend la liberté de n'avoir aucune religion), un motif de distinction illicite prévu par la Charte québécoise des droits de la personne. Cette exclusion, ajoute la Cour, " compromet son droit à l'exercice, en pleine égalité, de sa liberté de conscience et de religion ${ }^{18}$. La pause de deux minutes entre la récitation de la prière et le début de l'assemblée, loin d'atténuer le

14. Ibid., par. 78 .

15. Ibid., par. 64.

16. Ibid., par. 72.

17. Ibid., par. 77.

18. Ibid., par. 64. 
caractère religieux du règlement, au contraire l'accentue : "Cette solution qu'a adoptée le conseil, c'est-à-dire inviter les citoyens à quitter physiquement la salle le temps de la prière, fait ressortir l'effet d'exclusion de la prière ${ }^{19}$.

Pour conclure au caractère religieux de la pratique, le Tribunal des droits de la personne avait examiné les agissements du maire, des conseillers et le contexte de la récitation de la prière. À cet égard, deux des trois salles où les séances publiques se déroulent contiennent des symboles religieux : la statue du Sacré-Cour dans l'une et le crucifix dans l'autre. Alors que le Tribunal avait ordonné leur enlèvement, la Cour suprême, suivant en cela la Cour d'appel, a conclu que le Tribunal ne pouvait se prononcer sur les signes religieux en raison de l'absence d'enquête de la Commission des droits à ce sujet, la compétence du Tribunal étant tributaire de l'enquête menée par la Commission. Quant à la prière, la Cour suprême est d'avis que tant l'ancien texte que le nouveau constituent une « invocation à Dieu, lui attribuant les bénéfices accordés à la Ville et à ses citoyens, et lui demandant d'influencer les délibérations du conseil [municipal] ${ }^{20}$. Le signe de croix et la formule «Au nom du père, du fils et du Saint-Esprit » qui précède et termine la prière marquent le rituel religieux. De plus, les déclarations publiques du maire sont révélatrices de la fonction véritable de la pratique : "Ce combat-là, je le fais parce que j'adore le Christ. Quand je vais arriver de l'autre bord, je vais pouvoir être un peu orgueilleux. Je vais pouvoir lui dire: "Je me suis battu pour vous ; je suis même allé en procès pour vous". Il n'y a pas de plus bel argument. C'est extraordinaire. Ce combat-là, je le fais parce que j'adore le Christ, je veux aller au ciel et c'est le plus noble combat de toute ma vie. » De même, devant le Tribunal, le maire réitère ses propos et y ajoute : " Ce sont des choses que j'ai dites. Effectivement, si on insiste autant sur ça, c'est parce qu'on a la foi. C'est parce qu'on veut le manifester. C'est tout le conseil municipal qui est derrière moi. C'est bien entendu que ce n'est pas un combat strictement personnel. C'est tout le conseil municipal. Je suis mandaté. C'est parce que j'ai la foi et pour moi c'est la valeur la plus importante de toutes les valeurs que je peux avoir. »

Après avoir conclu que le règlement et la pratique de récitation de la prière contreviennent à l'obligation de neutralité religieuse de la Ville et que la prière crée une discrimination fondée sur la religion ayant pour effet de compromettre le droit de M. Simoneau à l'exercice, en pleine égalité, de sa liberté de conscience et de religion, la Cour suprême du Canada ordonne

19. Ibid., par. 101.

20. Ibid., par. 100 . 
que le règlement soit déclaré inopposable à M. Simoneau et que la Ville et son maire cessent de réciter la prière dans les salles de délibérations du conseil municipal. La Ville et son maire ont aussi été condamnés à verser à M. Simoneau des dommages-intérêts compensatoires de 15000 \$ et des dommages-intérêts punitifs de 15000 \$. 